IMMUNOTHERAPY

\title{
Nivolumab keeps HCC in check and opens avenues for checkmate
}

Annually, 750,000 new cases of hepatocellular carcinoma (HCC) are diagnosed worldwide, mostly at an advanced stage, with dismal outcomes. Sorafenib, the only approved systemic therapy for advanced-stage HCC, is of limited benefit, and no standard second-line therapy exists. New data indicate the therapeutic potential of immunotherapy with the anti-PD-1 antibody nivolumab in this disease.

In the phase I/II CheckMate 040 trial, 262 patients with advanced-stage HCC were treated with nivolumab; $76 \%$ had received prior systemic therapy, predominantly with sorafenib, and $68 \%$ had extrahepatic disease. In a dose-escalation cohort comprising 48 patients, only one dose-limiting toxicity was observed, and no maximum tolerated dose was defined. Among this group, the objective response rate (ORR) was $15 \%$, with three complete and four partial responses, and the disease control rate (DCR) was $58 \%$. The median overall survival duration was 15 months; at both 6 and 9 months, $66 \%$ of patients were alive.

In a dose-expansion cohort, 214 patients received nivolumab at a dose of $3 \mathrm{mg} / \mathrm{kg}$ every 2 weeks. The ORR was $20 \%$, comprising three complete and 39 partial responses, and the DCR was $64 \%$; 6-month and 9-month survival was $83 \%$ and $74 \%$, respectively. Of note, the ORRs were similar across groups stratified according to hepatitis B or C status, or sorafenib exposure, and were independent of PD-L1 expression level ( $<1 \%$ or $\geq 1 \%$ on tumour cells). To put these findings into perspective, the ORRs in previous trials of first-line sorafenib and second-line regorafenib were only 2-3\% and $7 \%$, respectively. Furthermore, the median overall survival with first-line sorafenib is typically $<11$ months.

Importantly, nivolumab was well tolerated, irrespective of hepatitis B/C infection, with no new safety signals. In the dose-expansion cohort, 40 patients
(19\%) had grade 3 or 4 adverse events, nine of which were classed as 'serious', and only 24 patients discontinued treatment owing to toxicity. Moreover, EQ-5D-3L index assessments at baseline and at 25 weeks revealed no significant changes in patient-reported health-related quality of life.

Although results of randomized comparisons are needed, these findings strongly suggest that nivolumab therapy, in the first or second line, can keep advanced-stage $\mathrm{HCC}$ in check. In addition, the good safety profile of nivolumab provides scope to achieve 'checkmate' with combination therapies, in both advanced and early disease settings. Notably, anti-CTLA-4 antibody therapy has promising efficacy in patients with HCC, warranting investigation of dual PD-1/CTLA-4 blockade, which has proven efficacy in other cancers.

David Killock therapy, in the first or second line, can keep advancedstage HCC in check...
ORIGINAL ARTICLE El-Khoueiry, A. B. et al. Nivolumab in patients with advanced hepatocellular carcinoma (CheckMate 040): an open-label, non-comparative, phase $1 / 2$ dose escalation and expansion trial. Lancet http://dx.doi.org/10.1016/S01406736(17)31046-2 (2017) 